sustaining AKT phosphorylation in early mouse embryos. Taken together, data obtained with kinase inhibitors and quantitative immunofluorescence analyses support strongly the idea that early mouse embryos inherit fully Ser472/ Thr308-phosphorylated AKT from oogenesis and that initial embryo cleavages are independent on growth factors, making PI3K and PDK1 activities dispensable. This conclusion is in agreement with previous observations that mouse preimplantation embryo development requires PI3K activity from the 8/16-cell stage. ${ }^{8}$

If TCL1 is not relevant to AKT phosphorylation in one-cell and two-cell embryos, is this factor needed for the phosphorylated AKT transfer to nucleus? We have addressed this issue by determining the intracellular distribution of Ser473/ Thr308-phosphorylated AKT in two-cell embryos that were genetically deficient in TCL1. Phosphorylated AKT had a striking cortical localization and was lacking in blastomere nuclei (Figure 1e), pinpointing the cell membrane as an obligatory step among the intracellular movements of phosphorylated AKT. In fact, it is commonly accepted that TCL1 oligomerizes with AKT at the level of cell membrane and that the TCL1-AKT complex is then transferred to nucleus. ${ }^{11}$

In conclusion, early mouse embryos display a physiological dissociation between the TCL1 functions of AKT phosphorylation and phosphorylated AKT transfer to nucleus, pinpointing the latter function as the essential one for the AKT-mediated promotion of cell proliferation. In addition, the present finding that TCL1 enters one-cell embryo pronuclei, while phosphorylated AKT does not, suggests that TCL1 also plays an AKTindependent role(s) at the beginning of embryo development. In light of the recent finding that TCL1 binds the PNPase exoribonuclease, an enzyme that specifically degrades poly$\mathrm{A}^{+} \mathrm{RNAs}^{12}$ it is tempting to hypothesize that this factor is also relevant for the degradation of maternally derived messages.
Acknowledgements. We thank Dr Domenico Grillo for help in quantitative immunofluorescence determinations and $\mathrm{Dr}$ Adriana Bosco for genotyping $\mathrm{TCl}^{-1-}$ mice. This work was supported by grants from Istituto Pasteur - Fondazione Cenci Bolognetti to FM and Associazione Italiana Ricerca sul Cancro and Ministero della Salute to GR.

\section{MT Fiorenza ${ }^{1,2}, S$ Torcia $^{1,2}$, S Canterini $^{1,2}$, A Bevilacqua ${ }^{1,2}$, MG Narducci ${ }^{3}$, G Ragone ${ }^{3}$, $\mathrm{CM} \mathrm{Croce}^{4}, \mathrm{G} \mathrm{Russo}^{3}$ and F Mangia*,1,2}

${ }^{1}$ Istituto Pasteur - Fondazione Cenci Bolognetti, Sapienza University, Rome 00185, Italy;

2 Section of Neuroscience, Department of Psychology, Sapienza University, Rome 00185, Italy;

3 Istituto Dermopatico dell'Immacolata, Istituto di Ricovero e Cura a Carattere Scientifico, Rome 00167, Italy and

4 Human Cancer Genetics Program, Department of Molecular Virology, Immunology, and Medical Genetics, OSU School of Medicine, and Comprehensive Cancer Center, Ohio State University, Columbus, $\mathrm{OH} 43210$, USA

* Corresponding author: Professor F Mangia, Department of Psychology, Section of Neuroscience, Sapienza University of Rome, Via dei Marsi 78, Roma 00185, Italy. Tel.: + 39-0649917784; Fax: + 39-0649917873;

E-mail: franco.mangia@uniroma1.it

\author{
1. Virgilio L et al. Proc Natl Acad Sci USA 1998; 95: 3885-3889. \\ 2. Matoba $\mathrm{R}$ et al. PLOS ONE 2006; 1: e26. \\ 3. Ivanova $\mathrm{N}$ et al. Nature 2006; 442: 533-538. \\ 4. Narducci MG et al. Proc Natl Acad Sci USA 2002; 99: 11712-11717. \\ 5. Kunstle G et al. Mol Cell Biol 2002; 22: 1513-1525. \\ 6. Pekarsky $Y$ et al. Proc Natl Acad Sci USA 2000; 97: 3028-3033. \\ 7. Mora A et al. Semin Cell Dev Biol 2004; 15: 161-170. \\ 8. Lu DP et al. J Cell Sci 2004; 117: 1567-7156. \\ 9. Sarbassov DD et al. Science 2005; 307: 1098-1101. \\ 10. Komander D et al. Structure 2004; 12: 215-226. \\ 11. Teitell MA. Nat Rev Cancer 2005; 5: 640-648. \\ 12. French SW et al. Cancer Lett 2006; 248: 198-210.
}

\title{
Autophagy promotes necrosis in apoptosis-deficient cells in response to ER stress
}

\author{
Cell Death and Differentiation (2008) 15, 422-425; doi:10.1038/sj.cdd.4402234; published online 5 October 2007
}

Dear Editor,

Disruption of endoplasmic reticulum (ER) function is associated with numerous human diseases. ${ }^{1,2}$ It leads to the initiation of a stress response known as the unfolded protein response, whose initial goal is to resolve the ensuing stress; however, when unable to do so, it induces cell death. ${ }^{3,4}$ ER stress-induced cell death has been shown to proceed primarily through apoptosis. It remained unclear whether ER stress is also associated with other forms of cell death. Using bax $^{-/-}$bak $^{-/-}$or $\mathrm{Bcl}-\mathrm{xL}$-overexpressing cells that are defective in apoptosis, here, we show that prolonged ER stress still results in cell death in a fashion resembling necrosis. This necrosis-like cell death is associated with autophagy. In response to ER stress, autophagy is induced in both wild-type and $\mathrm{bax}^{-/-} \mathrm{bak}^{-/-}$cells to a similar extent. Overexpression of wild-type Bax or Bak, as well as ERtargeted Bak does not induce autophagy, indicating that the multi-domain proapoptotic Bcl-2 proteins do not affect autophagy. Inhibition of autophagy results in enhanced cell 
death in apoptosis-competent cells, but reduced cell death in apoptosis-deficient cells. Thus, in response to ER stress, while autophagy serves as a survival response to delay apoptosis, it promotes cell death by necrosis in cells with impaired apoptosis.

It has been previously shown that cells deficient in both Bax and Bak are resistant to apoptosis induced by ER stress. ${ }^{5}$ As ER stress is often sustained for extended periods of time under physiological conditions, ${ }^{3,4}$ we sought to investigate cell survival in response to prolonged ER-stress treatment. Mouse embryonic fibroblasts (MEFs) isolated from wild-type and bax $^{-1-}$ bak $^{-1-}$ mice were treated with ER stressors thapsigargin, tunicamycin, and brefeldin A. Consistent with previous reports, almost all wild-type MEFs died after 1-2 days of ER stress treatment, while Bax/Bak doubly deficient cells were well protected. Interestingly, when treated with ER stress for a prolonged period of time, apoptosis-deficient $\mathrm{bax}^{-/-} \mathrm{bak}^{-/-}$ MEFs progressively died (Figure 1a). Similar results were observed in MEFs ectopically expressing the antiapoptotic protein Bcl-xL (Supplementary Figure S1A). Thus, ER stress can cause two phases of cell death, the first of which is Bax/ Bak-dependent apoptosis; the other is Bax/Bak-independent or Bcl-xL uninhibitable cell death that occurs at a slower rate.

Upon further examination using phase-contrast microscopy the dead bax $^{-/-}$bak $^{-/-}$cells displayed morphological features such as plasma membrane dilation and disruption that resemble necrosis (Supplementary Figure S1B). To characterize this more, we examined the cleavage of poly(ADPribose) polymerase (PARP) from its full-length $114 \mathrm{kDa}$ form to generate an $89 \mathrm{kDa}$ fragment, a characteristic feature of apoptotic cells. The caspase-3-specific $89 \mathrm{kDa}$ fragment was observed in wild-type cells 1-2 days after ER-stress treatment, but not in $\mathrm{bax}^{-1-} \mathrm{bak}^{-/-}$cells even after 3-4 days, at which time the amount of cell death was comparable to that of wild-type cells treated for 1-2 days (Figure 1b). Furthermore, the release of HMGB1 into the extracellular environment, an indicator of necrosis, ${ }^{6}$ was observed to coincide with the timing of ER stress-induced bax $^{-1-}$ bak $^{-1-}$ cell death, but not wild-type cell death (Supplementary Figure S1C). These findings indicate that while apoptosis is blocked in bax ${ }^{-/-}$ bak $^{-1-}$ cells, prolonged treatment with ER stress can induce caspase-independent, necrotic cell death.

When observed with an electron microscope, wild-type and bax $^{-1-}$ bak $^{-1-}$ MEFs exposed to ER stress for a short period of time (12 h) displayed an increase in multi- or double-layered membrane structures enclosing electron-dense materials, which are characteristic of autophagosomes (Figure 1c). The induction of autophagy was verified further by the conversion of microtubule-associated protein 1 (MAP1) light chain (LC3) from its cytosolic form (LC3-I) to a membrane-bound form (LC3-II) (Figure 1C, Supplementary Figure S2C and D), a key event in autophagy. ${ }^{7}$ 3-Methyladenine (3MA), a pharmacological inhibitor of autophagy, blocked the induction of GFP-LC3 puncta formation. In contrast, chloroquine, a chemical that blocks lysosome function by increasing lysosomal $\mathrm{pH}$, increased the formation of GFP-LC3 puncta (Supplementary Figure $\mathrm{S} 2 \mathrm{C}$ and $\mathrm{D}$ ). These findings indicate that ER stress may induce a autophagic response in both wild-type and bax ${ }^{-/}$ bak ${ }^{-1-}$ cells. We noticed that after tunicamycin treatment for $48 \mathrm{~h}$ wild-type cells appeared apoptotic, characterized by condensed chromatin, whereas $\mathrm{bax}^{-/-} \mathrm{bak}^{-/-}$MEFs showed no signs of apoptosis yet drastic accumulation of autophagosomes (Supplementary Figure S2A and B). This suggests that after longer exposure to ER stress, wild-type cells die by apoptosis if the damage is not relieved, whereas autophagy keeps accumulating in $b a x^{-/-} b^{-1-}$ cells due to their deficiency in apoptosis. The prolonged accumulation of autophagy may eventually lead to cell damage and demise independent of Bax and Bak.

Interestingly, it was noted that autophagy was induced in both wild-type and $\mathrm{bax}^{-/-} \mathrm{bak}^{-1-}$ cells in response to ER stress. The balance between the levels of the antiapoptotic and proapoptotic Bcl-2 family of proteins plays a critical role in determining the fate of a cell to survive or to die by apoptosis. The $\mathrm{BH} 1$ and $\mathrm{BH} 2$ domains of $\mathrm{Bcl}-2$ are required for Bcl-2-Bax interaction and inhibition of apoptosis. ${ }^{8}$ It has been recently reported that the antiapoptotic Bcl-2 family members can regulate autophagy, ${ }^{9-11}$ possibly by interacting with Beclin 1.9,10 Beclin 1 is essential for autophagy induction, ${ }^{12,13}$ and was found to interact with $\mathrm{Bcl}-2$ via the $\mathrm{BH} 1$ and $\mathrm{BH} 2$ domains of $\mathrm{Bcl}-2 .^{10}$ In light of these reports, there exists the possibility that the proapoptotic proteins Bax and Bak may affect the induction of autophagy via their interaction with $\mathrm{Bcl}-2$. To address this issue, we quantitated the formation of autophagosomes in wild-type and bax ${ }^{-/}$ bak $^{-1-}$ MEFs treated with ER stress. Both cell types showed equivalent amounts of GFP-LC3 puncta (Supplementary Figure S3A). In addition, both wild-type and $\mathrm{bax}^{-/-} \mathrm{bak}^{-/-}$ MEFs treated with ER stress showed clear conversion of LC3I to LC3-II, with no apparent difference, judged by immunoblotting of LC3 (Supplementary Figure S3B). The lysosomal protease inhibitors E-64-D and pepstatin $A^{14}$ enhanced the accumulation of LC3-II to a similar degree in wild-type and $\mathrm{bax}^{-/-} \mathrm{bak}^{-1-}$ cells (Supplementary Figure S3C and D). These results indicate that deficiency in Bax and Bak has no significant effect on ER stress-induced autophagy.

Conversely, we tested whether overexpression of Bak or Bax could affect the induction of autophagy. DsRed-tagged Bak or Bax were co-transfected with GFP-LC3 into bax ${ }^{-/}$ bak $^{-1-}$ MEFs. Bak was predominantly localized to mitochondria indicated by its punctate localization. Expression of DsRed-Bak did not induce autophagy, nor did it affect tunicamycin-induced autophagy (Figure 1d). Bax was predominantly localized in the cytosol. In response to ER stress, it translocated from the cytosol to mitochondria. Similar to Bak, expression of DsRed-Bax failed to induce autophagy and did not alter ER stress-induced autophagy (Figure 1d and Supplementary Figure S4A). An ER-targeted Bak mutant also failed to affect autophagy induction (Supplementary Figure S4C). This mutant has been previously shown to localize to the $\mathrm{ER}$ and cause $\mathrm{ER} \mathrm{Ca}^{2+}$ release and apoptosis. ${ }^{15}$ Taken together, our findings indicate that unlike the antiapoptotic Bcl-2 family proteins, the multi-domain proapoptotic Bcl-2 proteins Bax and Bak do not affect autophagy, nor do they affect Bcl-2's ability to regulate autophagy.

Autophagy has been shown to promote either cell survival or death. Given that autophagy can be induced by ER stress, we evaluated whether autophagy plays a role in bax $^{-/-}$bak $^{-/-}$cell death resulting from prolonged ER stress. 

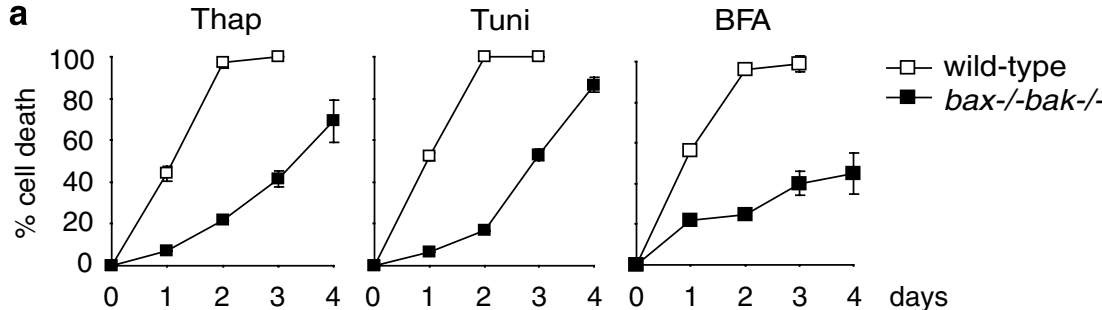

b

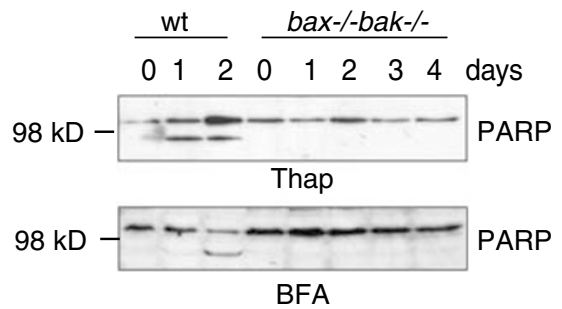

C

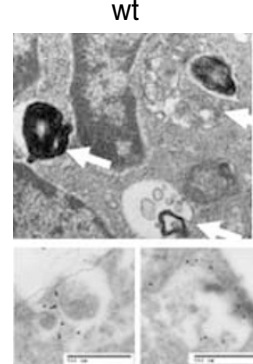

bax-/-bak-/-
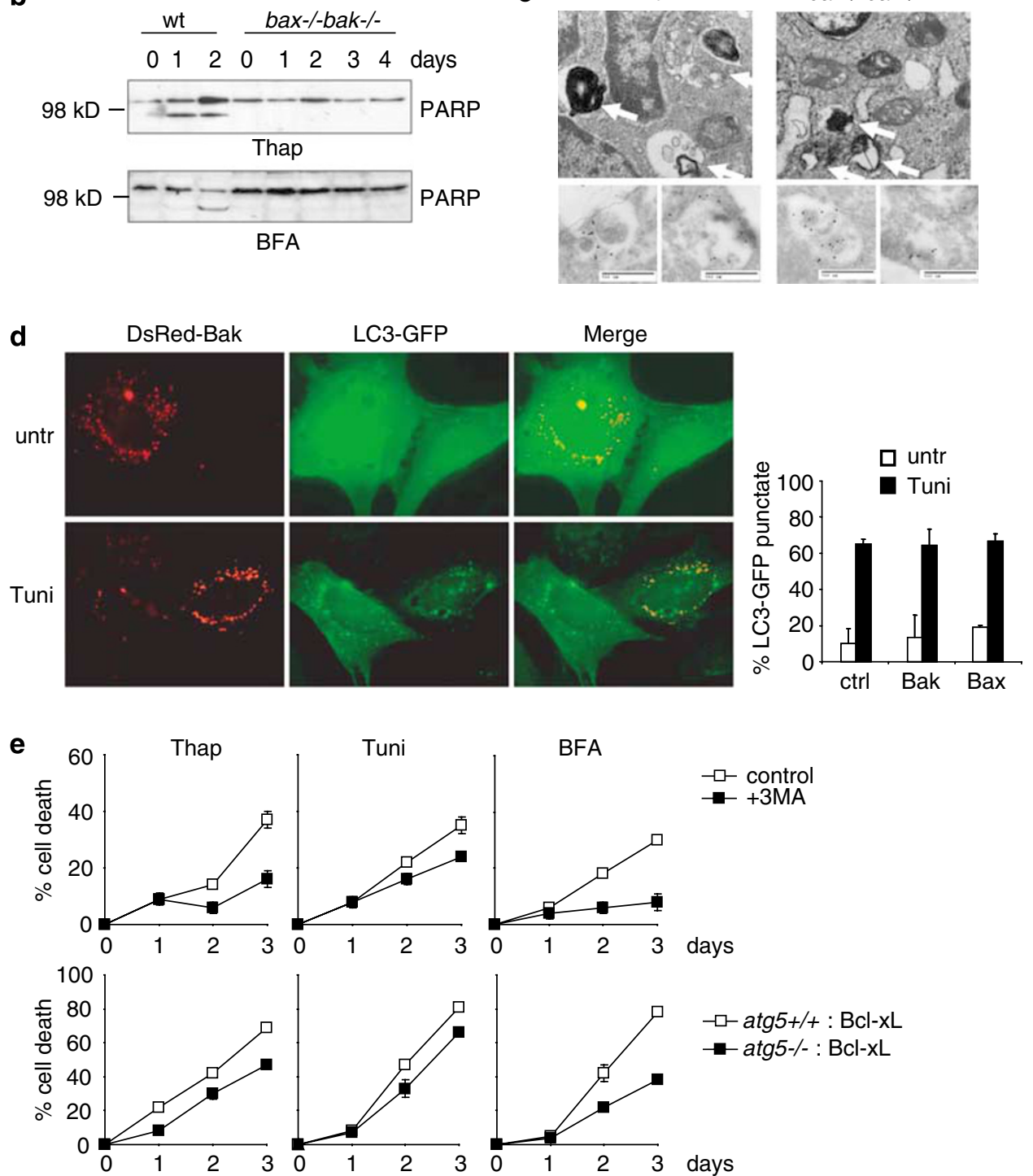

$-\square-\operatorname{atg} 5+/+: \mathrm{Bcl}-\mathrm{xL}$

$\rightarrow-\operatorname{atg} 5-/-: B c l-x L$

Figure 1 (a) ER stress can induce non-apoptotic cell death. Wild-type and $b a x^{-1-}$ bak ${ }^{-/-}$MEFs were treated with thapsigargin $(0.1 \mu \mathrm{M})$, tunicamycin $(0.5 \mu \mathrm{g} / \mathrm{ml})$, or brefeldin A $(0.5 \mu \mathrm{g} / \mathrm{ml})$. At the indicated time points, cells were collected and cell death was measured by PI exclusion. Data are averages of triplicate treatments \pm S.E.M. $\left(P<0.01\right.$ for all time points). (b) Caspase-3-dependent PARP cleavage does not occur in bax ${ }^{-1}$ bak $^{-/-}$MEFs. Wild-type and bax ${ }^{-1}$ bak $^{-1-}$ cells were treated with either brefeldin A or thapsigargin for the indicated times, cell lysates were prepared followed by immunoblotting for PARP. (c) ER stress induces autophagosome formation. Wild-type and $b_{a x}{ }^{-1}$ bak ${ }^{-1-}$ MEFs stably expressing GFP-conjugated LC3 were treated with tunicamycin $(0.5 \mu \mathrm{g} / \mathrm{ml})$ for $12 \mathrm{~h}$. Cells were then fixed and observed with an electron microscope and representative images are shown. Electron microscopy immunohistochemistry was also performed using an anti-GFP antibody. ${ }^{20}$ Note the presence of gold particles indicative of autophagosomes in both wild-type and bax ${ }^{-1}$ bak ${ }^{-1-}$ cells. Bar $=500 \mathrm{~nm}$. (d) Multi-domain proapoptotic Bcl-2 proteins do not affect autophagy. bax ${ }^{-1}$ bak $^{-1-}$ MEFs were transfected with GFP-LC3 together with DsRed-tagged Bak or Bax. Twenty-four hours later, cells were left untreated or treated with tunicamycin $(0.5 \mu \mathrm{g} / \mathrm{ml})$ for $8 \mathrm{~h}$. Cells were then observed under a Zeiss Axiovert fluorescence microscope. Note that DsRed-Bak did not induce autophagy, nor did it alter tunicamycininduced autophagy. Quantitation of autophagic cells in control cells and cells expressing DsRed-Bax or Bak. (e) Inhibition of autophagy protects apoptosis-deficient MEFs from ER stress-mediated cell death, while promoting death in apoptosis-competent cells. atg $5^{/-}$and $\operatorname{atg} 5^{+/+} \mathrm{MEFs}$ were infected with a retroviral construct expressing Bcl-xL. Cells were treated for the indicated times with ER stress and cell death was measured by PI exclusion and indicated as an average of triplicate samples + S.E.M. $(P<0.01$ for all time points). Note that $\operatorname{atg} 5^{-/}$cells died faster than atg $5^{+/+}$cells, whereas when Bcl-xL was expressed, atg $5^{/-}$cells died slower than atg $5^{+/+}$cells 
Cells treated with ER stress in the presence of the autophagy inhibitor 3MA appeared to be more adherent to the culture plates, and maintained relatively normal fibroblast appearance (Supplementary Figure S5A). In addition, when measured by plasma membrane permeability, 3MA significantly enhanced the viability of $\mathrm{bax}^{-/-} \mathrm{bak}^{-/-}$cells in response to ER stress (Figure 1e). These results indicate that autophagy can enhance cell death in $\mathrm{bax}^{-1-} \mathrm{bak}^{-1-}$ cells. In contrast, 3MA enhanced ER stress-induced cell death in apoptosis-competent wild-type cells (Supplementary Figure $\mathrm{S} 5 \mathrm{~B})$. These findings suggest that autophagy may have an opposite effect in determining cell fate in response to ER stress in apoptosis-competent and deficient cells. To determine more specifically whether autophagy plays a role in Bax/Bak-independent cell death in response to ER stress, the essential autophagy factor Atg5 was knocked down by short hairpin RNA in $\mathrm{bax}^{-/-} \mathrm{bak}^{-/-}$MEFs. Similar to 3MA, inhibition of autophagy by Atg5 knockdown resulted in significant protection against thapsigargin and brefeldin A (Supplementary Figure S5C).

The above results suggest that autophagy can promote cell death in apoptosis-deficient cells. To determine whether this is a peculiar effect that can only be observed in bax $^{-/-}$bak $^{-/-}$ cells, Bcl-xL was ectopically expressed at similar levels in atg $5^{+1+}$ and $\operatorname{atg} 5^{-1}$ cells to block apoptosis. When treated with ER stress, atg5 $5^{-/}$cells died faster than $a t g 5^{+/+}$cells (Supplementary Figure S5D), consistent with recent reports that autophagy acts as a survival mechanism to suppress apoptosis in response to ER stress. ${ }^{16,17}$ As anticipated, $\mathrm{Bcl}-\mathrm{xL}$ protected both atg $5^{+/+}$and atg $5^{-1}$ cells from acute apoptosis. In response to prolonged ER stress the Bcl-xLoverexpressing cells progressively died. Interestingly, $\mathrm{Bcl}-\mathrm{xL}$-overexpressing atg5 $5^{-}-\mathrm{MEFs}$ survived better than $\mathrm{Bcl}-\mathrm{xL}$-overexpressing atg $5^{+/+}$MEFs (Figure 1e). Taken together, our findings indicate that while autophagy serves as a survival mechanism in apoptosis-competent cells, those with defects in apoptosis can utilize autophagy as a means to promote non-apoptotic cell death in response to ER stress.

Our results show that cells deficient in apoptosis, due to either the lack of both Bax and Bak, or expression of Bcl-xL, can die in response to prolonged ER stress. This Bax/Bakindependent and $\mathrm{Bcl}-\mathrm{xL}$ unblockable cell death is necrotic, as revealed by morphological and biochemical criteria. This may be pathologically significant especially in cells with impaired apoptosis, such as cancer cells. Additionally, we find that ER stress-induced necrosis is associated with autophagy. While autophagy was originally described as a cell survival mechanism, it recently has been proposed that under certain circumstances it can be a mechanism of cell death. ${ }^{9,18}$ With respect to ER stress, it is suggested that autophagy helps the cell to adapt to the stress. Here, we show that while autophagy can be induced to similar levels in wild-type and bax $^{-/-}$bak $^{-/-}$ cells in response to ER stress, the resulting outcome of this response appears to be much different. While inhibition of autophagy using 3MA or by Atg5 knockdown was able to delay cell death in $b x^{-/-} b k^{-/-}$cells, inhibition of autophagy made apoptosis-competent cells more sensitive to ER stress.
Thus in wild-type cells, autophagy apparently confers protection from apoptosis resulting from excessive ER stress, possibly by promoting protein degradation to resolve the stressing conditions. ${ }^{16-19}$ In contrast, in apoptosis-deficient cells prolonged autophagy provokes cell death, and its absence imparts protection. This may be because in these cells where excessive ER stress fails to induce apoptosis, the stressing situation keeps accumulating to a point where autophagy is massively induced subsequently leading to cell damage and necrosis. It is interesting to note, however, inhibition of autophagy did not completely abrogate this Bax/ Bak-independent cell death, nor did it promote clonological survival (data not shown). This indicates that cell death in response to ER stress may involve complex cell death pathways. Autophagy, rather than acting as a distinguished form of cell death, instead contributes to the cell fate determination by differentially affecting apoptosis and necrosis.

Acknowledgements. We thank Drs. Tamotsu Yoshimori and Noboru Mizushima for providing reagents, Susan Van Horn (Central Microscopy Imaging Center at Stony Brook University) for assistance on electron microscopy. We also thank Drs. Michael Frohman, Nancy Reich, Howard Crawford, Richard Lin, Patrick Hearing, and Janet Hearing for critical readings. WXZ is supported by the NCl Howard Temin Award and the Carol Baldwin Breast Cancer Research Foundation at Stony Brook.

\section{E Ullman ${ }^{1,4}$, Y Fan ${ }^{2,4}$, M Stawowczyk ${ }^{1}$, H-M Chen ${ }^{1}$, $Z Y_{u} e^{3}$ and $W-X$ Zong $^{*, 2}$}

${ }^{1}$ Graduate Program in Molecular and Cellular Biology, Stony Brook University, Stony Brook, NY, USA;

${ }^{2}$ Department of Molecular Genetics and Microbiology, Stony Brook University, Stony Brook, NY, USA and

${ }^{3}$ Department of Neurology, Mount Sinai School of Medicine, New York, NY, USA

4 These authors have contributed equally to this work.

* Corresponding author: W-X Zong, Department of Molecular Genetics and Microbiology, Stony Brook University, 128 Life Sciences Building, Stony Brook, New York, NY 11794-5222, USA.

Tel: + 631632 4104; Fax: +631632 9797;

E-mail: wzong@notes.cc.sunysb.edu

1. Schroder M et al. Mutat Res 2005; 569: 29-63.

2. Marciniak SJ et al. Physiol Rev 2006; 86: 1133-1149.

3. Rao RV et al. Cell Death Differ 2004; 11: 372-380.

4. Boyce M et al. Cell Death Differ 2006; 13: 363-373.

5. Wei MC et al. Science 2001; 292: 727-730.

6. Scaffidi $P$ et al. Nature 2002; 418: 191-195.

7. Kabeya $Y$ et al. EMBO J 2000; 19: 5720-5728.

8. Yin XM et al. Nat Cell Biol 1994; 369: 321-323.

9. Shimizu $S$ et al. Nat Cell Biol 2004; 6: 1221-1228.

10. Pattingre $S$ et al. Cell 2005; 122: 927-939.

11. Hoyer-Hansen M et al. Mol Cell 2007; 25: 193-205.

12. Liang XH et al. Nature 1999; 402: 672-676.

13. Yue $Z$ et al. Proc Natl Acad Sci USA 2003; 100: 15077-15082.

14. Tanida I et al. Autophagy 2005; $1: 84-91$.

15. Zong WX et al. J Cell Biol 2003; 162: 59-69.

16. Ogata M et al. Mol Cell Biol 2006; 26: 9220-9231.

17. Kouroku $Y$ et al. Cell Death Differ 2007; 14: 230-239.

18. Yu L et al. Science 2004; 304: 1500-1502.

19. Ding WX et al. J Biol Chem 2007; 282: 4702-4710.

20. Wang QJ et al. J Neurosci 2006; 26: 8057-8068. 